\title{
A METHOD OF MEASURING FABRY-PAROT ETALON TRANSMITTANCE BY USING FREQUENCY COMB LASER
}

\author{
Yufei Chu ${ }^{1,2}$, Dong Liu ${ }^{1}$, Decheng Wu ${ }^{1}$, Zhenzhu Wang ${ }^{1}$, Qian Deng ${ }^{1,2}$, Zhiqiang Kuang ${ }^{1,2}$, Lu Li $^{1,2}$, \\ Peng Zhuang ${ }^{1,2}$ Zhiyuan Fang ${ }^{1,2}$, Chenbo Xie ${ }^{1,2}$, Yingjian Wang ${ }^{1,2}$
}

${ }^{1}$ Key Laboratory of Atmospheric optics, Anhui Institute of Optics and Fine Mechanics, Chinese Academy of Sciences, Hefei, Anhui 230031, China

${ }^{2}$ University of Science and Technology of China, Hefei, Anhui 230031, China

*Email:wyj@aiofm.ac.cn

\begin{abstract}
This paper presents a method for instantaneous measurement of Fabry-Parot(F-P) transmittance curve. Compared with the previous method, this method has many advantages, such as scanning-free, simple in structure and accuracy.
\end{abstract}

\section{INTRODUCTION}

The F-P etalon is an interferometer which mainly consisting by two flat glass or quartz plates. It can be used in high-resolution spectroscopy as a high-resolution optical filter or a precision wavelength meter. In laser systems, it is often used to narrow the linewidth in the cavity or to make the laser system operate in a single mode. It can be used as the medium bandwidth control and tuning device in broadband picosecond laser. It also be widely used in the lidar system for Doppler wind measurement and aerosol detection. Due to the very wide application of the F-P etalon, the standard transmission curve of the F-P is a very important parameter. The general F-P etalon measures the transmittance curve by tuning the wavelength of the laser source or tuning the angle of incident light. The F-P etalon generally measures the transmittance curve of the etalon by tuning the wavelength of the laser source. Depending on the bandwidth of the etalon, the laser source with wavelength tuning function is very expensive, and the FP etalon of wide free spectral range is difficult to find a corresponding laser source with a wide range of wavelength tuning. Moreover, both the tuning of the wavelength and the angle of incident light are nonlinear, and the measurement accuracy is insufficient. This paper proposes a new method for detecting the transmittance curve of a F-P etalon using a frequency comb laser source. Due to the equal spacing of the frequency of the frequency comb laser, the transmittance curve of the F-P etalon can be measured at one time. And the frequency interval and spectral range are tunable, which can avoid the above defects. This new method greatly reduces the cost of measuring transmission curve, improve measurement accuracy and effectiveness and has great theoretical and practical value.

This paper introduces the working principle of F-P etalon and common transmittance measurement method, then introduce the characteristics of the frequency comb laser source, finally introduce the method of measuring F-P transmittance based on frequency comb laser and its simulation results.

\section{METHODOLOGY}

\subsection{Working principle of F-P etalon and} common transmittance measurement method

A traditional etalon consists of two reflective surfaces that are parallel to each other. As shown in Figure 1, the amplitude reflectance of the two reflective surfaces are $R_{1}$ and $R_{2}$, respectively, and the refractive index of the medium between the two reflective surfaces is $n$, the thickness of the medium is $\mathrm{h}$.

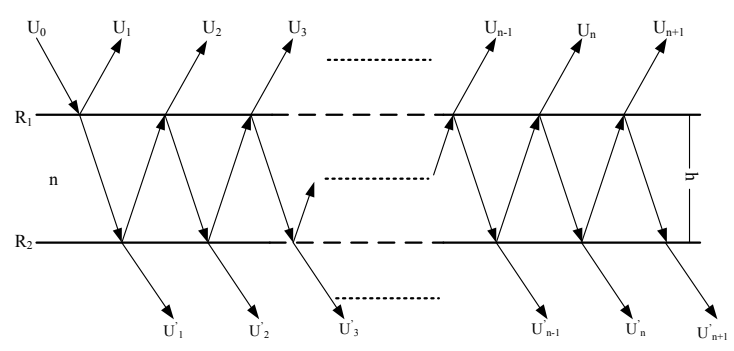

Figure 1 Principle of etalon transmittance

Transmittance of F-P etalon is defined as ${ }^{[1]}$ :

$$
T=\frac{U^{\prime} U^{* *}}{U_{0} U_{0}^{*}}=\frac{\left(1-R_{1}\right)\left(1-R_{2}\right)}{\left(1-R_{1} R_{2}\right)^{2}+4 \sqrt{R_{1} R_{2}} \sin ^{2}\left(\frac{\delta}{2}\right)}
$$


When the amplitude reflectance of the two surfaces of the etalon is equal, that is, $R_{1}=R_{2}$, and the intensity reflectance of each surface is $\mathrm{R}=\mathrm{R}_{1}{ }^{2}$. The transmittance of the etalon can be simplified as follows

$$
T=\frac{1}{1+\frac{4 R}{(1-R)^{2}} \sin ^{2}\left(\frac{\delta}{2}\right)}
$$

Which, $\delta=\frac{4 \pi n h \cos \theta}{\lambda}$, from the expression of $\mathrm{T}$ and $\delta$, we can get that the transmittance is related to the length of the cavity, the wavelength and the incident angle, etc. The transmittance curve can be obtained directly by changing the wavelength, or the transmittance change caused by the change of the corresponding wavelength can also be deduced by changing the length of the cavity or changing the incident angle ${ }^{[2]}$. However, no matter the angle or the length of the cavity or the wavelength of the laser is changed, there is no guarantee that the change is linear, which will introduce errors. Next section we will introduce a frequency comb laser source that can provide multiple frequency components at a time.

\subsection{The characteristic of the frequency comb laser source}

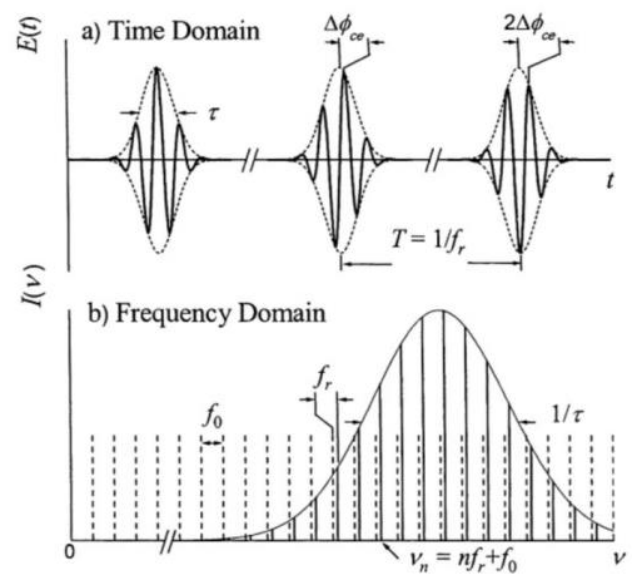

Figure 2 Standard comb: time (upper) and frequency (down) domain

In optics, a frequency comb is a laser source whose spectrum consists of a series of discrete, equally spaced frequency lines. Frequency combs can be generated by a number of mechanisms, including periodic modulation (in amplitude and/or phase) of a continuous-wave laser, fourwave mixing in nonlinear media, or stabilization of the pulse train generated by a mode-locked laser. Much work has been devoted to the latter mechanism, which was developed around the turn of the 21 st century and ultimately led to one half of the Nobel Prize in Physics being shared by John L. Hall and Theodor W. Hänsch in 2005. ${ }^{[3 \cdot 4]}$

The time and frequency characteristics of ultrashort laser pulses are shown in the figure 2 . Because the spectrum is very similar to the comb in daily life, it is called "optical frequency comb" ${ }^{[5]}$. The frequency comb light source is more expensive, so we can replace it by a similar frequency comb laser source ${ }^{[6]}$.

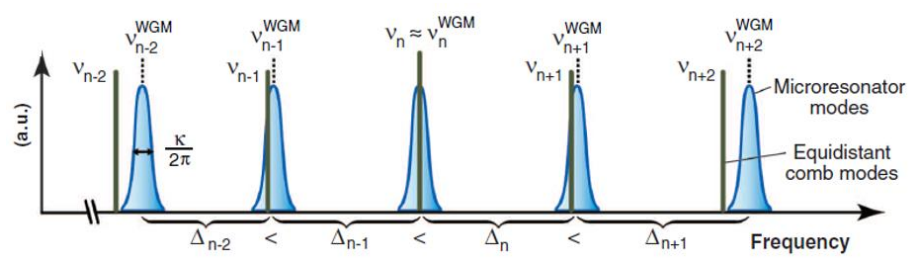

Figure 3 Whispering-gallery-mode Laser

The whispering gallery mode $\mathrm{e}^{[7]}$ is a typical similar frequency comb laser source which has multiple frequency components, but not equal intervals, as shown in Figure 3.

\subsection{Measuring F-P transmittance with frequency comb laser}

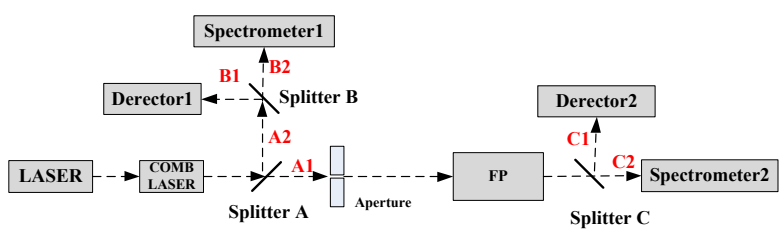

Figure 4. Schematic of measuring FP transmittance curve using frequency comb light source

The seed laser generates a variety of frequency components through a frequency comb laser, and passes through splitter A, then beam would be divided into beam A1 and A2. The beam $\mathrm{A} 2$ is divided into beam $\mathrm{B} 1$ and $\mathrm{B} 2$ by splitter $\mathrm{B}$. The beam B2 is detected by the spectrometer 1 and the beam B1 is received by the detector 1 . The beam A1 pass through the F-P etalon, and is divided into beam $\mathrm{C} 1$ and $\mathrm{C} 2$ by splitter $\mathrm{C}$. Beam $\mathrm{C} 2$ is detected by the spectrometer 2 and beam $\mathrm{C} 1$ is received by the detector 2 . By comparing the 
relative changes of the corresponding frequency components before and after the F-P, the transmittance of the corresponding frequency can be obtained, and the transmission curve can be obtained by connecting all the frequency components.

\section{RESULTS}

\subsection{Parameters of the F-P etalon}

The main technical parameters of solid state etalon for aerosol detection are given in the table 1 .

\begin{tabular}{|c|c|}
\hline \multicolumn{2}{|c|}{ Table 1: Main parameters of FP etalons } \\
\hline Parameters & Index \\
\hline Wavelength(nm) & 532 \\
\hline Refractive index & 1.461 \\
\hline Beam divergence full angle(mrad) & 8 \\
\hline Thickness(mm) & 0.1311 \\
\hline Optical aperture(mm) & 40 \\
\hline Effective aperture (mm) & 30 \\
\hline Reflectivity & $86 \%$ \\
\hline Surface accuracy @633nm) & $\lambda / 100$ \\
\hline Fine number & 17.9 \\
\hline Peak transmittance & 0.860 \\
\hline FWHM(pm) & 41.3 \\
\hline Center wavelength (nm) & 532.12 \\
\hline Free spectral range (pm) & 739 \\
\hline
\end{tabular}

From the parameters in the table, we can get Numerical simulation results of etalon transmittance curves:

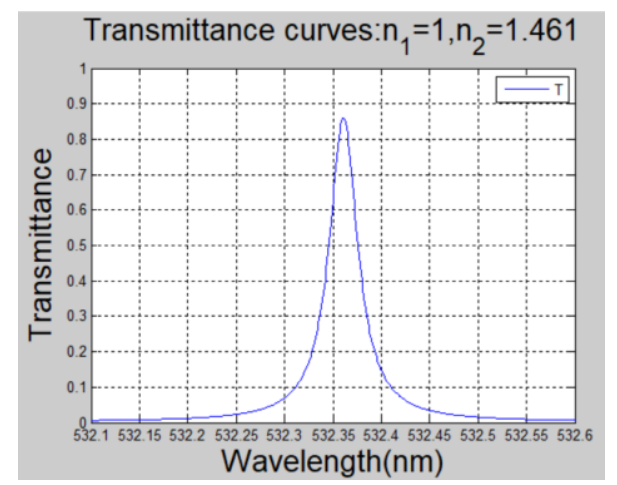

Figure 5 Numerical simulation results of solid state etalon transmittance curves
From the above numerical simulation results, when the thickness of the etalon is 131 um and the two optical surfaces are plated with $86 \%$ of the reflective film, the center wavelength of the etalon is $532.37 \mathrm{~nm}$, and the peak transmittance is $86 \%$.

\subsection{Simulation results}

We use the method introduced in section 2.3 to test the passing rate curve of F-P.

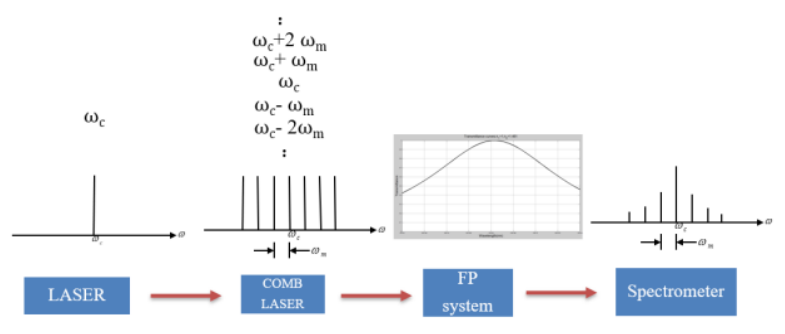

Figure 6 Numerical simulation results of solid state etalon transmittance curves

As we know in section 2.1, the different frequency components have different transmission rates for F-P etalon. According to the different frequency components in Figure 4, When the different frequency components pass through the F-P etalon, the rate of change of frequency will be consistent with the rate of transmission.

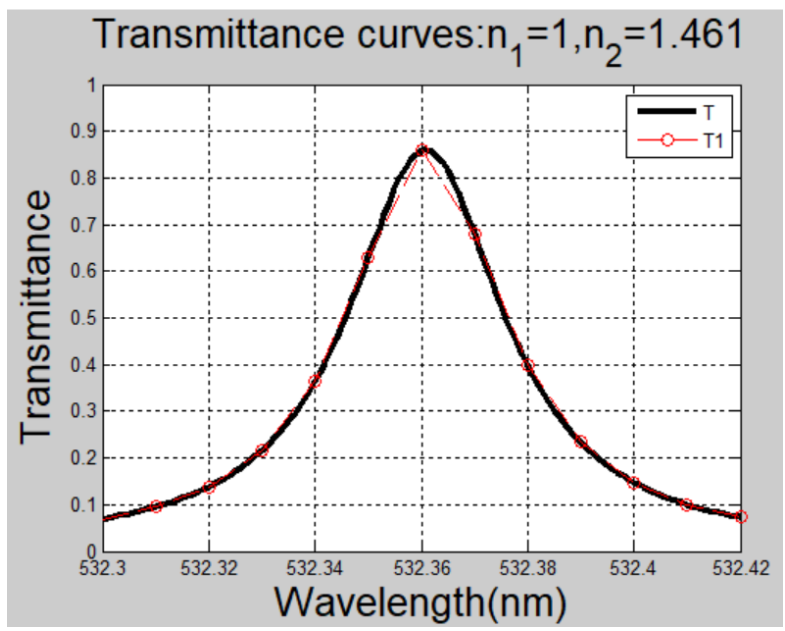

Figure 7 Theoretical transmittance curve comparison

$\mathrm{T}$ represents the theoretical pass rate curve, and $\mathrm{T} 1$ represents the transmittance curve obtained by the method described in this paper. As can be seen from the figure, the match is very good. 


\section{ACKNOWLEDGEMENTS}

This work was supported by the Youth Innovation Promotion Association CAS (2017482) and by the general propgram of National Natural Science Foundation of China (41875033). Key Program for External Cooperation of Bureau of International Cooperation, Chinese Academy of Sciences (GJHZ201976) is also acknowledged.

\section{REFERENCES}

[1] Shen F H., et al. Wind lidar based on Fizeau interferometer [J]. Infrared and Laser Engineering, 2006, 35(6): 691-695 .

[2] Sun D S., et al. Performance analysis of direct detection Doppler lidar for wind measurement[J]. Infrared and Laser Engineering, 2003, 32(2): 115-117

[3] Hall J L. Nobel Lecture: Defining and measuring optical frequencies[J]. Review of Modern Physics, 2006, 78(4):1279-1295.

[4] Hänsch T W. Nobel Lecture: Passion for precision[J]. Review of Modern Physics, 2006, 78(78):1297-1309.

[5] Ye J, Cundiff S T. Femtosecond Optical Frequency Comb: Principle, Operation, and Applications[M]. 2005.

[6]Chu YF., etal. Fluorescence radiation characteristics based on evanescent wave pumping in a microfluidic chip [J]. Acta Physica Sinica, 2017(10):156-162.

[7] Zhang Y X., et al. Threshold characteristics of evanescent-wave pumped whispering-gallery-mode fiber laser [[J]. Acta Physica Sinica, 2009, 58(5). 